\title{
Treinamento de resistência em escolares: Uma Revisão Integrativa
}

\author{
Resistance training in schools: An integrative review \\ Entrenamiento de resistencia en escolares: Una revisión integradora
}

Recebido: 09/03/2021 | Revisado: 17/03/2021 | Aceito: 18/03/2021 | Publicado: 26/03/2021

\author{
Nilson Vieira Pinto \\ ORCID: https://orcid.org/0000-0001-6548-8586 \\ Instituto Federal de Educação, Ciência e Tecnologia do Ceará, Brasil \\ E-mail: nilsonvieira@ifce.edu.br \\ Rayssa Camurça Pereira \\ ORCID: https://orcid.org/0000-0001-9340-6103 \\ Instituto Federal de Educação, Ciência e Tecnologia do Ceará, Brasil \\ E-mail: rayssacamurca2203@gmail.com
}

\begin{abstract}
Resumo
Diversos estudos têm discutido os benefícios e as estratégias de segurança para o treinamento de resistência em crianças e adolescentes, entretanto, a sua inserção nas aulas de educação física escolar ainda não é significativa. A análise das atuais evidências científicas sobre o treinamento de resistência em escolares pode ampliar a compreensão de sua ação pedagógica e possibilitar inserção deste conteúdo na escola. Nesse sentido, este estudo de revisão teve por objetivo sintetizar as evidências sobre o treinamento de resistência em escolares. As bases eletrônicas consultadas foram BVS, PUBMED e SCIELO, utilizando a combinação dos termos relativos ao treinamento de resistência, adolescentes e escola. $\mathrm{O}$ mapeamento resultou em 08 artigos, incluindo pesquisas de campo e revisões, publicados entre 2010 e 2020. Os resultados evidenciaram a melhora da aptidão física, da composição corporal e de variáveis bioquímicas, a redução de desvios posturais e do risco de lesões, bem como de doenças associadas ao sedentarismo, além de ampliar a motivação e a adesão dos escolares nas aulas de educação física. Estes resultados apontam que o treinamento de resistência, quando sob supervisão adequada, pode promover inúmeros benefícios e ampliar a motivação dos escolares.
\end{abstract}

Palavras-chave: Treinamento de resistência; Adolescente; Educação física e treinamento.

\begin{abstract}
Several studies have discussed the benefits and safety strategies for resistance training in children and adolescents, however, their inclusion in school physical education classes is still not significant. The analysis of the current scientific evidence on resistance training in schoolchildren can broaden the understanding of their pedagogical action and allow insertion of this content in school. In this sense, this review study aimed to synthesize the evidence on resistance training in schoolchildren. The electronic databases consulted were BVS, PUBMED and SCIELO, using the combination of terms related to resistance training, adolescents and school. The mapping resulted in 08 articles, including field research and reviews, published between 2010 and 2020. The results showed an improvement in physical fitness, body composition and biochemical variables, a reduction in postural deviations and the risk of injuries, as well as diseases associated with sedentary lifestyle, in addition to increasing the motivation and adherence of students in physical education classes. These results indicate that resistance training, when under adequate supervision, can promote numerous benefits and increase students' motivation.
\end{abstract}

Keywords: Resistance training; Adolescent; Physical education and training.

\section{Resumen}

Varios estudios han discutido los beneficios y estrategias de seguridad del entrenamiento de resistencia en niños y adolescentes, sin embargo, su inclusión en las clases de educación física escolar aún no es significativa. El análisis de la evidencia científica actual sobre el entrenamiento de resistencia en escolares puede ampliar la comprensión de su acción pedagógica y permitir la inserción de este contenido en la escuela. En este sentido, este estudio de revisión tuvo como objetivo sintetizar la evidencia sobre el entrenamiento de resistencia en escolares. Las bases de datos electrónicas consultadas fueron BVS, PUBMED y SCIELO, utilizando la combinación de términos relacionados con el entrenamiento de resistencia, adolescentes y escuela. El mapeo resultó en 08 artículos, incluyendo investigaciones de campo y revisiones, publicados entre 2010 y 2020. Los resultados mostraron una mejora en la condición física, composición corporal y variables bioquímicas, una reducción de las desviaciones posturales y el riesgo de lesiones, así como enfermedades asociadas. con sedentarismo, además de incrementar la motivación y adherencia de los estudiantes a las clases de educación física. Estos resultados indican que el entrenamiento de resistencia, cuando está bajo la supervisión adecuada, puede promover numerosos beneficios y aumentar la motivación de los estudiantes.

Palabras clave: Entrenamiento de resistencia; Adolescente; Educación y entrenamiento físico. 


\section{Introdução}

O treinamento de resistência (TR), também chamado de treinamento de força, é considerado um componente pedagógico do treinamento esportivo que visa o ganho de força, potência e/ou resistência muscular (Stricker, Faigenbaum \& McCambridge, 2020). Sua inserção nos programas de condicionamento físico para adolescentes tem se tornado cada vez mais comum no ambiente escolar.

Com a crescente popularidade e consequente aumento do número de adolescentes praticantes de musculação nas academias, torna-se cada vez mais importante a busca pelo entendimento pedagógico que leve a sua aplicabilidade com segurança e à promoção da saúde na escola.

Diversos estudos têm apontado evidências exitosas oriundas da prática regular do TR em adolescentes, apontando a melhora na aptidão física, do perfil lipídico e glicêmico, no controle ponderal, no aumento da resistência a lesões e na saúde mental, além de ampliar a adesão ao exercício físico (Behm, Faigenbaum, Falk \& Klentrou, 2008; Ughini, Becker \& Pinto, 2011; Benedet et al., 2013; Ruas, Brown \& Pinto, 2014).

Por ser uma modalidade que pode necessitar de alto investimento financeiro na aquisição e na manutenção dos equipamentos, tem sido um conteúdo pouco explorado na escola, o que repercute em uma módica análise reflexiva sobre sua abordagem pedagógica e consequentemente na exígua produção científica, geralmente limitada apenas pela adoção do conteúdo teórico sobre a musculação devido a ausência deste laboratório de prática pedagógica.

Contudo, o TR não deve se restringir apenas ao uso de aparelhos e tem apresentado inúmeros benefícios com o uso do peso corporal e/ou pesos livres (Menegon et al., 2016; Lesinski, Prieske \& Granacher, 2016). O desvelar científico sobre o TR em escolares pode ampliar a compreensão de sua ação pedagógica e possibilitar a inserção deste conteúdo na escola.

Desta forma, este artigo de revisão tem como objetivo analisar a produção científica atual sobre o TR em escolares procurando identificar as evidências produzidas em torno deste componente curricular neste ambiente de ensino.

\section{Metodologia}

Neste artigo utiliza-se a revisão integrativa como percurso metodológico, caracterizada pela análise e síntese de resultados dos estudos científicos sobre uma determinada questão, no intuito de sistematizar e organizar seus achados e ampliar a reflexão sobre o tema (Mendes, Silveira \& Galvão, 2008).

Esta revisão integrativa foi sistematizada em seis etapas distintas e fundamentais: a) definição da questão de pesquisa; b) busca na literatura científica; c) categorização das pesquisas mapeadas; d) avaliação dos resultados; e) interpretação dos resultados; e f) apresentação dos resultados da revisão integrativa (Mendes, Silveira e Galvão, 2008; Souza, Silva \& Carvalho, 2010).

Desta maneira, realizou-se buscas por artigos originais disponíveis nas bases de dados Biblioteca Virtual em Saúde (BVS), Public Medline (PubMED) e Scientific Electronic Library Online (SCIELO). No mapeamento, utilizou-se a combinação de descritores exatos e sinonímias validados no sítio da DECS (Descritores em Ciências da Saúde).

Os critérios de inclusão utilizados foram: (a) estudos que avaliem o treinamento de resistência/musculação em adolescentes publicados entre janeiro de 2010 a dezembro de 2020; (b) pesquisas experimentais ou revisões bibliográficas; (c) disponibilidade nos idiomas portugueses ou inglês; (d) estar indexado nas bases BVS, PUBMED ou SCIELO.

Em relação aos trabalhos excluídos desta revisão, destacam-se: resumos, teses, dissertações, monografias, artigos duplicados, pesquisas com mais de 10 anos de publicação, experimentos com animais e artigos que não estabelecem uma relação entre o treinamento resistido/musculação em escolares.

O levantamento bibliográfico, realizado no período entre 20 de dezembro de 2020 e 20 de fevereiro de 2021, destacou os seguintes elementos: amostra, objetivos utilizados, metodologia empregada e principais resultados. A Tabela 1 apresenta os 
resultados do mapeamento, os descritores utilizados e as bases de dados consultadas.

Tabela 1: Descritores de busca por base de dados utilizada no mapeamento e resultados.

\begin{tabular}{clccc}
\hline Base De Dados & \multicolumn{1}{c}{ Descritores } & Resultados & Achados & \%* $^{*}$ \\
\hline BVS & Musculação AND adolescentes & 1356 & 4 & 0,29 \\
& Resistance training AND school & 877 & 1 & 0,11 \\
\multirow{2}{*}{ PUBMED } & Resistance training AND teenagers & 2744 & 1 & 0,03 \\
& Resistance training AND teenagers AND school & 1318 & 1 & 0,07 \\
SCIELO & Musculação AND escola & 1 & 1 & 100 \\
Total & & $\mathbf{6 2 9 6}$ & $\mathbf{8}$ & $\mathbf{0 , 1 2 \%}$ \\
\hline
\end{tabular}

*Porcentagem de achados em relação ao total de resultados. Fonte: Elaboração própria com base no mapeamento realizado nas bases de dados.

A trajetória analítica de revisão teve início com uma triagem dos estudos nas bases eletrônicas de dados, sendo eliminados aqueles que não se relacionavam com a temática em questão e/ou atendessem aos critérios de inclusão previamente estabelecidos.

De um total de 6.296 estudos mapeados, o PUBMED apresentou a maior quantidade de resultados (2.744 trabalhos) e a BVS em número de achados (4 trabalhos). Deste total, apenas oito $(0,12 \%)$ atenderam aos critérios de inclusão e por sua vez, foram analisados na íntegra.

Na seção seguinte, apresentaremos o resultado da análise destes achados, evidenciando as atuais discussões sobre o TR em escolares. Nesse caminhar epistemológico, faz-se necessário estabelecer relações entre os elementos de discussão com os principais autores de referência.

\section{Resultados e Discussão}

Com base nos critérios de inclusão e exclusão foram selecionados 08 artigos científicos, disponíveis na BVS (04 trabalhos), PUBMED (03 trabalhos), e SCIELO (01 trabalho), incluindo estudos experimentais e revisões.

O artigo selecionado mais antigo foi publicado no ano de 2010, enquanto o mais recente, em 2020. Percebe-se uma distribuição dos estudos entre os anos de 2010 a 2020, tendo os anos de 2016 e 2020, o maior número de achados, o que denota uma perspectiva de novos estudos voltados ao TR em escolares. As revistas de indexação variam entre os extratos A1 e B3, com maior incidência para revistas com o qualis A (A1: 03 trabalhos; A2: 02 trabalhos), indicando uma maior qualidade nas produções científicas em torno desta temática.

O Quadro 1 apresenta informações detalhadas deste mapeamento (autores, ano de publicação, revista indexada, base indexada, título do trabalho e classificação no sistema Qualis/CAPES da revista), os quais relacionam treinamento de resistência, adolescentes e escola. 
Quadro 1: Detalhamento dos achados no mapeamento.

\begin{tabular}{|c|c|c|c|c|c|}
\hline Autores & Ano & $\begin{array}{c}\text { Revista } \\
\text { Indexada }\end{array}$ & $\begin{array}{c}\text { Base } \\
\text { Indexada }\end{array}$ & Título & Qualis* \\
\hline Velez et al. & 2010 & $\begin{array}{l}\text { Journal of } \\
\text { Strength and } \\
\text { Conditioning } \\
\text { Research }\end{array}$ & BVS & $\begin{array}{l}\text { The impact of a 12- } \\
\text { week resistance } \\
\text { training program on } \\
\text { strength, body } \\
\text { composition, and self- } \\
\text { concept of Hispanic } \\
\text { adolescents }\end{array}$ & A1 \\
\hline Rosa Santos et al. & 2020 & $\begin{array}{l}\text { Medicina } \\
\text { (Kaunas) }\end{array}$ & BVS & $\begin{array}{c}\text { Effects of } 12 \text { Weeks of } \\
\text { Resistance Training on } \\
\text { Cardiovascular Risk } \\
\text { Factors in School } \\
\text { Adolescents } \\
\end{array}$ & B2 \\
\hline Kennedy et al. & 2018 & $\begin{array}{l}\text { Medicine and } \\
\text { science in } \\
\text { sports and } \\
\text { exercise }\end{array}$ & BVS & $\begin{array}{c}\text { Implementing } \\
\text { Resistance Training in } \\
\text { Secondary Schools: A } \\
\text { Cluster Randomized } \\
\text { Controlled Trial }\end{array}$ & A1 \\
\hline $\begin{array}{c}\text { Stricker, Faigenbaum, } \\
\text { Mccambridge }\end{array}$ & 2020 & $\begin{array}{l}\text { Pediatrics } \\
\text { (Evanston) }\end{array}$ & BVS & $\begin{array}{l}\text { Resistance Training for } \\
\text { Children and } \\
\text { Adolescents. }\end{array}$ & $\mathrm{A} 2$ \\
\hline $\begin{array}{l}\text { Lesinski, Prieske, } \\
\text { Granacher }\end{array}$ & 2016 & $\begin{array}{l}\text { British journal } \\
\text { of sports } \\
\text { medicine }\end{array}$ & PUBMED & $\begin{array}{l}\text { Effects and dose- } \\
\text { response relationships } \\
\text { of resistance training } \\
\text { on physical } \\
\text { performance in youth } \\
\text { athletes: a systematic } \\
\text { review and meta- } \\
\text { analysis }\end{array}$ & A1 \\
\hline $\begin{array}{l}\text { Ruivo, Pezarat-Correia, } \\
\text { Carita }\end{array}$ & 2017 & $\begin{array}{l}\text { Journal of } \\
\text { Manipulative } \\
\text { and } \\
\text { Physiological } \\
\text { Therapeutics }\end{array}$ & PUBMED & $\begin{array}{l}\text { Effects of a Resistance } \\
\text { and Stretching Training } \\
\text { Program on Forward } \\
\text { Head and Protracted } \\
\text { Shoulder Posture in } \\
\text { Adolescents }\end{array}$ & $\mathrm{A} 2$ \\
\hline Benedet et al. & 2013 & $\begin{array}{l}\text { ABCS Health } \\
\text { Sciences }\end{array}$ & PUBMED & $\begin{array}{c}\text { Treinamento resistido } \\
\text { para crianças e } \\
\text { adolescentes }\end{array}$ & B3 \\
\hline Menegon et al. & 2016 & $\begin{array}{l}\text { Revista } \\
\text { Brasileira de } \\
\text { Ciências do } \\
\text { Esporte }\end{array}$ & SCIELO & $\begin{array}{c}\text { Musculação na } \\
\text { educação física escolar: } \\
\text { uma experiência no } \\
\text { ensino médio noturno }\end{array}$ & B1 \\
\hline
\end{tabular}

*Área de avaliação: Educação Física. Fonte: Elaboração própria.

Com vistas na exploração dos conteúdos mapeados e na análise qualitativa dos dados publicados, apresenta-se no Quadro 2 um esquema das informações coletadas que nortearão em sequência, a narrativa sistematizada destas informações. 
Quadro 2: Conteúdo das pesquisas mapeadas.

\begin{tabular}{|c|c|c|c|c|c|}
\hline Autor E Ano & Amostra & Objetivo & $\begin{array}{c}\text { Desenho Do } \\
\text { Estudo }\end{array}$ & Método & Resultado \\
\hline $\begin{array}{l}\text { VELEZ Et Al. } \\
\qquad(2010)\end{array}$ & $\begin{array}{c}\text { Adolescentes } \\
\text { hispânicos } \\
\text { (28: } 16 \hat{\bigcirc}^{\lambda} ; \\
12 \text {; }) \\
\text { (14 a } 18 \text { anos) }\end{array}$ & $\begin{array}{l}\text { Examinar os efeitos } \\
\text { de um programa de } \\
\text { treinamento de } \\
\text { resistência } \\
\text { estruturado na força, } \\
\text { composição } \\
\text { corporal e } \\
\text { autoconceito em } \\
\text { adolescentes } \\
\text { hispânicos normais } \\
\text { e com sobrepeso. }\end{array}$ & $\begin{array}{l}\text { Pesquisa de } \\
\text { campo }\end{array}$ & $\begin{array}{c}\text { Ensaio } \\
\text { clínico } \\
\text { controlado } \\
\text { / Estudo } \\
\text { de } \\
\text { avaliação }\end{array}$ & $\begin{array}{c}\text { O grupo experimental teve: } \\
-\uparrow \text { de força para supino } \\
\text { reto, remada sentada, } \\
\text { supino de ombro e } \\
\text { agachamento; } \\
-\downarrow \% \text { gordura corporal; } \\
-\uparrow \text { resistência, adequação } \\
\text { corporal atraente e } \\
\text { autovalor global. } \\
\text { O grupo controle teve: } \\
\text { - } \uparrow \text { ligeiro da \% de gordura } \\
\text { corporal. }\end{array}$ \\
\hline $\begin{array}{l}\text { Rosa Santos } \\
\text { et al. }(2020)\end{array}$ & $\begin{array}{c}\text { Adolescentes } \\
\left(122: 52{ }^{\gamma} ;\right. \\
70 \text {; }) \\
\text { (13 a } 16 \text { anos) }\end{array}$ & $\begin{array}{l}\text { Avaliar os efeitos da } \\
\text { aplicação de um } \\
\text { programa de } \\
\text { treinamento } \\
\text { resistido de } 12 \\
\text { semanas sobre os } \\
\text { fatores de risco } \\
\text { cardiovascular em } \\
\text { adolescentes. }\end{array}$ & $\begin{array}{l}\text { Pesquisa de } \\
\text { campo }\end{array}$ & $\begin{array}{l}\text { Estudo de } \\
\text { etiologia / } \\
\text { Estudo } \\
\text { observaci } \\
\text { onal / } \\
\text { Fatores de } \\
\text { risco }\end{array}$ & $\begin{array}{c}\downarrow \downarrow \text { nas variáveis de } \\
\text { triglicerídeos, lipoproteínas } \\
\text { de baixa densidade (LDL), } \\
\text { lipoproteínas de alta } \\
\text { densidade (HDL), glicose } \\
\text { no sangue e pressão arterial } \\
\text { sistólica. } \\
-\uparrow \text { da variável peso } \\
\text { corporal. }\end{array}$ \\
\hline $\begin{array}{c}\text { Kennedy et } \\
\text { al. } \\
(2018)\end{array}$ & $\begin{array}{c}\text { Adolescentes } \\
(607: 50.1 \% \\
\text { 우 } 14.1 \pm 0.5 \\
\text { anos) }\end{array}$ & $\begin{array}{c}\text { Examinar o efeito } \\
\text { de uma intervenção } \\
\text { escolar com foco no } \\
\text { treinamento de } \\
\text { resistência (TR) } \\
\text { para adolescentes. }\end{array}$ & $\begin{array}{l}\text { Pesquisa de } \\
\text { campo }\end{array}$ & $\begin{array}{l}\text { Ensaio } \\
\text { clínico } \\
\text { controlado } \\
\text { randomiza } \\
\text { do por } \\
\text { cluster. }\end{array}$ & $\begin{array}{c}\text { Após } 6 \text { meses foram } \\
\text { observados efeitos na parte } \\
\text { superior do corpo, mas não } \\
\text { na inferior, efeitos de } \\
\text { intervenção para } \\
\text { competência de habilidade } \\
\text { de TR e autoeficácia. } \\
\text { Com } 12 \text { meses foram } \\
\text { observados efeitos no IMC } \\
\text { de alunos com sobrepeso / } \\
\text { obesidade. }\end{array}$ \\
\hline $\begin{array}{c}\text { Stricker, } \\
\text { Faigenbaum, } \\
\text { Mccambridge } \\
\text { (2020) }\end{array}$ & $\begin{array}{c}\text { Crianças e } \\
\text { adolescentes }\end{array}$ & $\begin{array}{l}\text { Discutir os } \\
\text { benefícios, riscos e } \\
\text { recomendações } \\
\text { relacionadas ao } \\
\text { treinamento de } \\
\text { resistência para } \\
\text { crianças e } \\
\text { adolescentes. }\end{array}$ & Relatório clínico & $\begin{array}{l}\text { Estudos } \\
\text { experimen } \\
\text { tais }\end{array}$ & $\begin{array}{l}\text { O TR traz melhoria na } \\
\text { saúde, condicionamento } \\
\text { físico, reabilitação e } \\
\text { redução de lesões e } \\
\text { alfabetização física se as } \\
\text { atividades forem realizadas } \\
\text { com ênfase na técnica } \\
\text { adequada e bem } \\
\text { supervisionadas. }\end{array}$ \\
\hline $\begin{array}{l}\text { Lesinski, } \\
\text { Prieske, } \\
\text { Granacher } \\
\text { (2016) }\end{array}$ & $\begin{array}{c}43 \text { estudos } \\
\text { Jovens atletas } \\
\text { (6 a } 18 \text { anos) }\end{array}$ & $\begin{array}{l}\text { Quantificar os } \\
\text { efeitos específicos } \\
\text { de idade, sexo, } \\
\text { esporte e tipo de } \\
\text { treinamento do } \\
\text { treinamento de } \\
\text { resistência sobre o } \\
\text { desempenho físico, } \\
\text { e caracterizar as } \\
\text { relações dose- } \\
\text { resposta dos } \\
\text { parâmetros de } \\
\text { treinamento de }\end{array}$ & $\begin{array}{l}\text { Revisão de } \\
\text { literatura }\end{array}$ & $\begin{array}{c}\text { Revisão } \\
\text { sistemátic } \\
\text { a e meta- } \\
\text { análise de } \\
\text { estudos de } \\
\text { intervençã } \\
\quad \text { o }\end{array}$ & $\begin{array}{l}\text { Foram revelados efeitos } \\
\text { moderados do treinamento } \\
\text { de resistência na força } \\
\text { muscular e no desempenho } \\
\text { do salto vertical, e } \\
\text { pequenos efeitos no sprint } \\
\text { linear, agilidade e } \\
\text { desempenho específico do } \\
\text { esporte. Um período de } \\
\text { treinamento > } 23 \text { semanas, } \\
5 \text { séries / exercício, } 6-8 \\
\text { repetições / série, uma } \\
\text { intensidade de treinamento }\end{array}$ \\
\hline
\end{tabular}




\begin{tabular}{|c|c|c|c|c|c|}
\hline & & $\begin{array}{c}\text { resistência que } \\
\text { poderiam } \\
\text { maximizar os } \\
\text { ganhos no } \\
\text { desempenho físico } \\
\text { em atletas jovens. }\end{array}$ & & & $\begin{array}{l}\text { de } 80-89 \% \text { de } 1 \text { repetição } \\
\text { máxima }(\mathrm{RM}) \text { e } 3-4 \text { min } \\
\text { de descanso entre as séries } \\
\text { foram mais eficazes para } \\
\text { melhorar a força muscular. }\end{array}$ \\
\hline $\begin{array}{l}\text { Ruivo, } \\
\text { Pezarat- } \\
\text { Correia, } \\
\text { Carita } \\
(2017)\end{array}$ & $\begin{array}{c}\text { Adolescentes } \\
\text { portugueses } \\
\left(130: 48 \jmath^{\top} ; 82\right. \\
\text { ㅇ) } \\
(15 \text { a } 17 \text { anos) }\end{array}$ & $\begin{array}{l}\text { Avaliar os efeitos de } \\
\text { um programa de } \\
\text { treinamento de } \\
\text { resistência e } \\
\text { alongamento de } 16 \\
\text { semanas aplicado } \\
\text { em aulas de } \\
\text { educação física } \\
\text { sobre a postura } \\
\text { anterior da cabeça e } \\
\text { a postura do ombro } \\
\text { alongado em } \\
\text { adolescentes } \\
\text { portugueses. }\end{array}$ & $\begin{array}{c}\text { Pesquisa de } \\
\text { campo }\end{array}$ & $\begin{array}{c}\text { Estudo } \\
\text { prospectiv } \\
\text { o, } \\
\text { randomiza } \\
\text { do e } \\
\text { controlado }\end{array}$ & $\begin{array}{l}\text { - } \uparrow \text { nos ângulos cervicais e } \\
\text { do ombro foi observado no } \\
\text { grupo de intervenção do } \\
\text { pré-teste ao pós-teste. } \\
\text { - Para os escores de dor no } \\
\text { ombro em ambos os } \\
\text { grupos, não houve } \\
\text { mudanças significativas. }\end{array}$ \\
\hline $\begin{array}{c}\text { Benedet et al. } \\
\text { (2013) }\end{array}$ & $\begin{array}{c}25 \text { artigos } \\
\text { Crianças e } \\
\text { adolescentes }\end{array}$ & $\begin{array}{l}\text { Fazer uma análise } \\
\text { retrospectiva sobre } \\
\text { a evolução das } \\
\text { recomendações do } \\
\text { treinamento } \\
\text { resistido para } \\
\text { crianças e } \\
\text { adolescentes. }\end{array}$ & $\begin{array}{l}\text { Revisão de } \\
\text { literatura }\end{array}$ & $\begin{array}{l}\text { Estudo de } \\
\text { revisão }\end{array}$ & $\begin{array}{c}\text { - A literatura atual } \\
\text { recomenda a prática do TR } \\
\text { para jovens sob prescrição } \\
\text { e supervisão adequada } \\
\text { - Os achados apontam para } \\
\text { uma maior preocupação } \\
\text { com a segurança e a } \\
\text { aplicabilidade do TR no } \\
\text { início dos anos 90, } \\
\text { evoluindo para questões } \\
\text { voltadas a qualificação dos } \\
\text { profissionais e relações à } \\
\text { manutenção, diversificação } \\
\text { e aderência entre os jovens. }\end{array}$ \\
\hline $\begin{array}{l}\text { Menegon et } \\
\text { al. (2016) }\end{array}$ & $\begin{array}{c}19 \text { alunos } \\
(12 \hat{\jmath} ; 7 \text { + }) \\
\text { (17 a } 22 \text { anos) }\end{array}$ & $\begin{array}{l}\text { Planejar, aplicar e } \\
\text { avaliar a unidade de } \\
\text { ensino } \\
\text { "musculação" junto } \\
\text { a uma turma do } 3^{\circ} \\
\text { ano do ensino } \\
\text { médio noturno de } \\
\text { uma escola pública } \\
\text { estadual de } \\
\text { Maringá-PR. }\end{array}$ & $\begin{array}{l}\text { Pesquisa de } \\
\text { campo }\end{array}$ & $\begin{array}{l}\text { Estudo se } \\
\text { caracteriz } \\
\text { a como } \\
\text { um relato } \\
\text { de } \\
\text { experiênci } \\
\text { a de } \\
\text { caráter } \\
\text { qualitativo } \\
\text { e apoiado } \\
\text { por dados } \\
\text { quantitativ } \\
\text { os }\end{array}$ & $\begin{array}{c}\text { Alunos demonstraram } \\
\text { interesse e motivação em } \\
\text { praticar a musculação no } \\
\text { ambiente extraescolar e em } \\
\text { estudar e vivenciar novos } \\
\text { conteúdos nas aulas de } \\
\text { educação física a partir da } \\
\text { experiência vivida. }\end{array}$ \\
\hline
\end{tabular}

Legenda: $\widehat{\jmath}$ : homem; + $:$ mulher; $\uparrow:$ aumento; $\downarrow$ : diminuição. Fonte: Elaboração própria.

Em uma relação metodológica foram selecionadas cinco pesquisas de campo, duas revisões de literatura e um relatório clínico. Dos oito trabalhos selecionados apenas dois encontram-se publicados em língua portuguesa, sendo os demais em língua inglesa, o que evidencia uma predominância de estudos internacionais que discutem o TR em escolares.

De uma maneira geral, os estudos trazem evidências relacionadas aos benefícios da prática regular de TR em escolares, com ênfase no controle ponderal, na melhora da aptidão física, na prevenção da inatividade física e doenças associadas, na redução do risco de lesões, na melhora do perfil glicêmico e lipídico, na redução de desvios posturais e no 
aumento da motivação e consequente adesão a prática de exercícios físicos, decorrentes da prescrição e supervisão adequada do profissional de educação física em suas aulas.

Benedet et al., (2013), faz uma análise retrospectiva sobre a evolução das recomendações do TR para crianças e adolescentes. Nesta revisão de literatura, pontua que no início dos anos de 1990 a maior preocupação dos autores estava em esclarecer as questões de segurança e aplicabilidade do TR para crianças e adolescentes. Posteriormente, passam a ser abordadas questões voltadas para a qualificação dos profissionais, segurança e saúde da criança e do adolescente associadas ao TR, e mais recentemente, questões associadas à manutenção, diversificação e aderência do TR para os jovens.

Velez et al., (2010), enfatizam que o TR não é apenas seguro para os escolares, como tem sido recomendado para a promoção da saúde e desempenho do condicionamento físico, destacando que esta prática, quando sob supervisão adequada, promove a melhoria da composição corporal, da aptidão física e do autoconceito, revelando neste estudo que isso ocorre independente da atual composição corporal do aluno.

O estudo de Kennedy et al., (2018), demonstrou melhorias imediatas e sustentadas na aptidão muscular da parte superior do corpo, ampliação das competências e das habilidades relacionadas ao TR, bem como na motivação espontânea para a prática de atividade física. Observando ainda, uma redução da composição corporal dos alunos com sobrepeso/obesos.

Alguns autores têm demonstrado que programas multidisciplinares de atividade física são capazes de promover a redução ponderal em crianças e adolescentes obesos (Dao et al., 2004; Suskind et al., 2000; Barbosa \& Pinto, 2020), onde o TR tem contribuido para a prevenção da inatividade física e doenças associadas, como dislipidemia, hipertensão, diabetes, obesidade e outros fatores associados ao aparecimento de doenças cardíacas (Damas et al., 2018; Cichocki et al., 2017; Tomeleri et al., 2015; Arazi, Ghiasi \& Asgharpoor, 2013).

Corroborando, o estudo de Rosa Santos et al., (2020) avaliou 122 escolares que foram submetidos a um programa de TR nas aulas de educação física escolar durante 12 semanas, apresentando melhora significativa nos níveis glicêmicos, pressóricos e lipídicos dos alunos, caracterizando o TR como uma estratégia pedagógica positiva na promoção da saúde e no controle e/ou redução do risco em desenvolver doenças crônico-degenerativas, um achado que tem sido discutido por diversos autores (Lee \& Yoon, 2018; Piñeros-Garzón \& Rodríguez-Hernández, 2019; Andreis \& Balbé, 2018; Silva \& Martins, 2017; Barros et al., 2020).

Além disso, o TR em adolescentes pode ampliar o desempenho de habilidades motoras, os ganhos em velocidade e potência, o desenvolvimento de alfabetização física e a redução do risco de lesões (Stricker, Faigenbaum \& McCambridge, 2020) além de prevenir o desenvolvimento de diversas doenças crônico-degenerativas na vida adulta (Rosa Santos et al., 2020).

Diversos autores têm pontuado que o TR realizado com ênfase na técnica adequada e bem supervisionado, ao promover o ganho de aptidão física, amplia as habilidades motoras gerais e específicas e reduz o risco de lesões. Nota-se um novo olhar para a prescrição do TR em crianças e adolescentes que anteriormente preocupava-se com as possíveis lesões associadas ao treinamento e atualmente evoca-se a preocupação com lesões e outros eventos adversos ocasionadas pela falta de força adequada para acompanhar as demandas do treinamento (Lauersen, Andersen \& Andersen, 2018; Faigenbaum et al., 2016; Fröberg, Alricsson \& Ahnesjö, 2014).

O estudo de Ruivo, Pezarat-Correia e Carita (2017) aponta que um programa de exercícios resistidos, realizado duas vezes por semana e integrado às aulas de educação física escolar durante um período de 16 semanas, pode resultar na melhora da postura, com aumentos nos ângulos cervicais e dos ombros. Neste estudo, observou-se que após o período de 4 meses, houve um aumento significativo nos ângulos cervicais e do ombro nos alunos que praticaram o TR orientado, enquanto no grupo controle não houve diferenças significativas no ângulo cervical, sugerindo que a prática orientada do TR contribuiu para a melhora da postura destes adolescentes. 
Nesta trajetória, alguns estudos epidemiológicos relatam uma alta prevalência de desvios posturais da coluna em crianças e adolescentes (van Niekerk et al., 2008; Detsch et al., 2007) associada a dor musculoesquelética autorreferida (Diepenmaat et al., 2006) podendo estar relacionada a fatores psicossociais (Prins, Crous \& Louw 2008), a ergonomia (Murphy, Buckle \& Stubbs 2004) e/ou desequilíbrios de força e postura do pescoço e ombros (Brink et al., 2009). Desta forma, a inserção do TR nas aulas de educação física escolar pode possibilitar a prevenção ou a redução dos desvios posturais em escolares.

Vale destacar que, para além da promoção da saúde fundamentada nos parágrafos anteriores, a comunidade científica tem apontado que em comparação com as atividades aeróbicas, o TR pode ser uma atividade física mais atraente para os adolescentes. Em adição, Kennedy et al., (2018) destacaram que o TR é uma das poucas atividades em que os jovens com sobrepeso ou obesidade podem superar seus pares mais magros, devido a uma maior massa magra e força absoluta, além de favorecer um clima motivacional nas aulas capaz de promover a autoeficácia, capaz de ativar um mecanismo central de mudança de comportamento possibilitando uma maior adesão dos alunos nas aulas de educação física.

É interessante pontuar que estamos refletindo sobre um conteúdo ainda inabitual nas aulas de educação física na escola, mas que, sem dúvida, desperta um enorme interesse em ser vivenciado. Menegon et al., (2016) avaliaram o planejamento e a aplicação da unidade de ensino "musculação" junto a uma turma do $3^{\circ}$. ano do ensino médio noturno de uma escola pública estadual de Maringá-PR. Na fase diagnóstica deste estudo, apenas os esportes coletivos foram citados como o único trato pedagógico presente nas aulas de educação física, uma relação ainda hegemônica como aponta Bracht (2000). Entretanto, demonstraram um enorme interesse e motivação pela introdução deste conteúdo nas aulas e participaram ativamente dos encontros teórico-práticos realizados.

A inserção do TR nas aulas de educação física escolar possibilita o desenvolvimento de habilidades, conhecimentos e valores importantes para promover um estilo de vida saudável para além dos anos escolares (Kennedy et al., 2018) podendo ser uma excelente estratégia pedagógica capaz de aumentar os níveis diários de atividade espontânea em idade escolar (Stricker, Faigenbaum \& McCambridge, 2020).

Por fim, destaca-se que todos os estudos analisados evidenciam a necessidade de um treinamento físico realizado sob supervisão adequada e instrução qualificada para alcançar os objetivos e benefícios oriundos da prática regular. Existem inúmeras variáveis que contribuem para um programa de TR bem projetado, incluindo qualidade de instrução, ambiente de treinamento, frequência de treinamento, idade de treinamento, tipo de resistência usada, intensidade de esforço, número de séries e repetições, intervalo de descanso entre conjuntos e exercícios e duração do treinamento que devem ser considerados com responsabilidade (Malina, 2006; Faigenbaum et al., 2016; Moro, Bianco, Faigenbaum \& Paoli 2014; Faigenbaum, Lloyd \& Myer 2013).

Nesta trajetória, a revisão sistemática elaborada por Lesinski, Prieske e Granacher (2016) destaca que programas de TR com pesos livres são eficazes para aumentar a força muscular em jovens. Ressalta-se ainda que este estudo procurou analisar os efeitos específicos da idade, sexo, esporte e tipo de treinamento sobre o desempenho físico de jovens, apontando que o melhor resultado no desempenho físico está relacionado ao TR com menos repetições e intensidades mais altas.

É interessante acrescentar que o TR nas aulas de educação física escolar não deve se limitar apenas a levantar pesos. Para além disso, deve incluir uma ampla gama de movimentos com o peso corporal que podem ser utilizados com segurança e são capazes de promover a melhora na aptidão física relacionada á saúde e ao desempenho esportivo destes escolares (Stricker, Faigenbaum \& McCambridge, 2020).

\section{Considerações Finais}

Os benefícios e as estratégias pedagógicas mais seguras para o treinamento de resistência em crianças e adolescentes 
tem sido evidenciado pela comunidade cientifica, entretanto, as possibilidades de intervenção no ambiente escolar ainda permanecem com pouca expressão científica.

Foram destacados o controle ponderal, a melhora da aptidão física, a prevenção do sedentarismo e de doenças associadas, a redução do risco de lesões, a melhora do perfil glicêmico e lipídico, a redução de desvios posturais e o aumento da motivação e consequente adesão a prática de exercícios físicos, decorrentes da prescrição e supervisão adequada nas aulas de educação física.

As evidências nos direcionam ao entendimento de que o treinamento de resistência, quando sob supervisão adequada, é uma estratégia pedagógica segura, capaz de promover inúmeros benefícios e ampliar a motivação dos escolares nas aulas de educação física. Que a sua ação pedagógica não está limitada ao uso de equipamentos, devendo ampliar as possibilidades de intervenção neuromuscular de acordo com as habilidades e competências de cada turma.

Nota-se a necessidade de mais estudos que ampliem a compreensão pedagógica do treinamento de resistência em escolares e que sensibilizem o profissional de educação física a inserir este conteúdo nas suas aulas de forma segura e com resultados significativos aos seus alunos.

\section{Referências}

Andreis, N. G., \& Balbé, G. P. (2018). A influência do treinamento de força em indivíduos com síndrome metabólica. Biomotriz, 12(1).

Arazi, H., Ghiasi, A., \& Asgharpoor, S. (2013). A comparative study of cardiovascular responses to two rest intervals between circuit resistance exercises in normotensive woman. Revista Brasileira de Medicina do Esporte, 19(3), 176-180.

Barbosa, L. A., \& Pinto, N. V. (2020). Aptidão física relacionada à saúde em escolares praticantes de ginástica coletiva. Research, Society and Development, 9(5), e141953351-e141953351.

Barros, A. M. G., Visco, D. B., da Silva, R. M. P., da Paiva Alves, S. P., da Silva Simões, M. O., Medeiros, C. C. M., \& de Carvalho, D. F. (2020). Perfil lipídico em crianças com sobrepeso e obesidade: uma revisão integrativa. Research, Society and Development, 9(11), e4349119952-e4349119952.

Behm, D. G., Faigenbaum, A. D., Falk, B., \& Klentrou, P. (2008). Canadian Society for Exercise Physiology position paper: resistance training in children and adolescents. Applied physiology, nutrition, and metabolism, 33(3), 547-561.

Benedet, J., Freddi, J. C., Luciano, A. P., Almeida, F. D. S., Silva, G. L. D., Hinnig, P. D. F., \& Adami, F. (2013). Treinamento resistido para crianças e adolescentes. ABCS health sci.

Bracht, V. (2000). Esporte na escola e esporte de rendimento. Movimento (ESEFID/UFRGS), 6(12).

Brink, Y., Crous, L. C., Louw, Q. A., Grimmer-Somers, K., \& Schreve, K. (2009). The association between postural alignment and psychosocial factors to upper quadrant pain in high school students: a prospective study. Manual therapy, 14(6), 647-653.

Cichocki, M., Fernandes, K. P., Castro-Alves, D. C., \& Gomes, M. V. D. M. (2017). Atividade física e modulação do risco cardiovascular. Revista Brasileira de Medicina do Esporte, 23(1), 21-25.

Damas, F., Libardi, C. A., Ugrinowitsch, C., Vechin, F. C., Lixandrão, M. E., Snijders, T., ... \& Phillips, S. M. (2018). Early-and later-phases satellite cell responses and myonuclear content with resistance training in young men. PloS one, 13(1), e0191039.

Dao, H. H., Frelut, M. L., Oberlin, F., Peres, G., Bourgeois, P., \& Navarro, J. (2004). Effects of a multidisciplinary weight loss intervention on body composition in obese adolescents. International Journal of Obesity, 28(2), 290-299.

Detsch, C., Luz, A. M. H., Candotti, C. T., Oliveira, D. S. D., Lazaron, F., Guimarães, L. K., \& Schimanoski, P. (2007). Prevalência de alterações posturais em escolares do ensino médio em uma cidade no Sul do Brasil. Revista Panamericana de Salud Publica, 21, 231-238.

Diepenmaat, A. C. M., Van der Wal, M. F., De Vet, H. C. W., \& Hirasing, R. A. (2006). Neck/shoulder, low back, and arm pain in relation to computer use, physical activity, stress, and depression among Dutch adolescents. Pediatrics, 117(2), 412-416.

Faigenbaum, A. D., Lloyd, R. S., MacDonald, J., \& Myer, G. D. (2016). Citius, Altius, Fortius: efeitos benéficos do treinamento resistido para jovens atletas: revisão narrativa. British Journal of Sports Medicine, 50 (1), 3-7.

Faigenbaum, A. D., Lloyd, R. S., \& Myer, G. D. (2013). Youth resistance training: past practices, new perspectives, and future directions. Pediatric exercise science, 25(4), 591-604.

Fröberg, A., Alricsson, M., \& Ahnesjö, J. (2014). Awareness of current recommendations and guidelines regarding strength training for youth. International journal of adolescent medicine and health, 26(4), 517-523.

Kennedy, S. G., Smith, J. J., Morgan, P. J., Peralta, L. R., Hilland, T. A., Eather, N., \& Lubans, D. R. (2018). Implementing resistance training in secondary schools: a cluster randomized controlled trial. Medicine \& Science in Sports \& Exercise, 50(1), 62-72. 
Lee, E. Y., \& Yoon, K. H. (2018). Epidemic obesity in children and adolescents: risk factors and prevention. Frontiers of medicine, 12(6), 658-666.

Lauersen, J. B., Andersen, T. E., \& Andersen, L. B. (2018). Strength training as superior, dose-dependent and safe prevention of acute and overuse sports injuries: a systematic review, qualitative analysis and meta-analysis. British journal of sports medicine, 52(24), 1557-1563.

Lesinski, M., Prieske, O., \& Granacher, U. (2016). Effects and dose-response relationships of resistance training on physical performance in youth athletes: a systematic review and meta-analysis. British journal of sports medicine, 50(13), 781-795.

Malina, R. M. (2006). Weight training in youth-growth, maturation, and safety: an evidence-based review. Clinical journal of sport medicine, 16(6), 478-487.

Mendes, K. D. S., Silveira, R. C. D. C. P., \& Galvão, C. M. (2008). Revisão integrativa: método de pesquisa para a incorporação de evidências na saúde e na enfermagem. Texto \& contexto-enfermagem, 17(4), 758-764.

Menegon, D., Kocourek, G. D., da Silva Lima, S. B., Lima, W. F., Kravchychyn, C., \& de Oliveira, A. A. B. (2016). Musculação na educação física escolar: uma experiência no ensino médio noturno. Revista Brasileira de Ciências do Esporte, 38(2), 171-178.

Moro, T., Bianco, A., Faigenbaum, A. D., \& Paoli, A. (2014). Pediatric resistance training: current issues and concerns. Minerva pediatrica, 66(3), $217-227$.

Murphy, S., Buckle, P., \& Stubbs, D. (2004). Classroom posture and self-reported back and neck pain in schoolchildren. Applied ergonomics, 35(2), 113-120.

Piñeros-Garzón, F. S., \& Rodríguez-Hernández, J. M. (2019). Factores de riesgo asociados al control glucémico y síndrome metabólico en pacientes con diabetes mellitus tipo 2. Villavicencio, Colombia. Universidad y Salud, 21(1), 61-71.

Prins, Y., Crous, L., \& Louw, Q. A. (2008). A systematic review of posture and psychosocial factors as contributors to upper quadrant musculoskeletal pain in children and adolescents. Physiotherapy theory and practice, 24(4), 221-242.

Rosa Santos, L. D., Araujo, S. S. D., Vieira, E. F. D. S., Estevam, C. D. S., Santos, J. L. D., Wichi, R. B., \& Marçal, A. C. (2020). Effects of 12 weeks of resistance training on cardiovascular risk factors in school adolescents. Medicina, 56(5), 220.

Ruas, C. V., Brown, L. E., \& Pinto, R. S. (2014). Treinamento de força para crianças e adolescentes: adaptações, riscos e linhas de orientação. Brazilian Journal of Motor Behavior, 8(1), 1-9.

Ruivo, R. M., Pezarat-Correia, P., \& Carita, A. I. (2017). Effects of a resistance and stretching training program on forward head and protracted shoulder posture in adolescents. Journal of manipulative and physiological therapeutics, 40(1), 1-10.

Silva, D. A. S., \& Martins, P. C. (2017). Impact of physical growth, body adiposity and lifestyle on muscular strength and cardiorespiratory fitness of adolescents. Journal of bodywork and movement therapies, 21(4), 896-901.

Souza, M. T. D., Silva, M. D. D., \& Carvalho, R. D. (2010). Revisão integrativa: o que é e como fazer. Einstein (São Paulo), 8(1), $102-106$.

Stricker, P. R., Faigenbaum, A. D., \& McCambridge, T. M. (2020). Resistance training for children and adolescents. Pediatrics, 145(6).

Suskind, R. M., Blecker, U., Udall, Jr, J. N., Von Almen, T. K., Schumacher, H. D., Carlisle, L., \& Sothern, M. S. (2000). Recent advances in the treatment of childhood obesity. Pediatric diabetes, 1(1), 23-33.

Tomeleri, C. M., Ronque, E. R., Silva, D. R., Júnior, C. G. C., Fernandes, R. A., Teixeira, D. C., \& Cyrino, E. S. (2015). Prevalence of dyslipidemia in adolescents: comparison between definitions. Revista Portuguesa de Cardiologia, 34(2), 103-109.

Ughini, C. C., Becker, C., \& Pinto, R. S. (2011). Treinamento de força em crianças: segurança, benefícios e recomendações. Conexões, 9(2), 177-197.

Van Niekerk, S. M., Louw, Q., Vaughan, C., Grimmer-Somers, K., \& Schreve, K. (2008). Photographic measurement of upper-body sitting posture of high school students: a reliability and validity study. BMC musculoskeletal disorders, 9(1), 1-11.

Velez, A., Golem, D. L., \& Arent, S. M. (2010). The impact of a 12-week resistance training program on strength, body composition, and self-concept of Hispanic adolescents. The Journal of Strength \& Conditioning Research, 24(4), 1065-1073. 